\title{
High resolution electron microscopy as a tool for structural investigations
}

\author{
G SINGH and R S RAI \\ Department of Physics, Banaras Hindu University, Varanasi 221005 , India.
}

\begin{abstract}
Lattice imaging technique has been used to study an unknown high period polytype structure of $\mathrm{SiC}$. A known structure like $6 \mathrm{H}$ has been studied by tilted beam twodimensional images to determine optimum conditions for deriving structural information. The technique has then been used to determine a new structure $4411 \mathrm{R}$ and its intergrowth structures), and the lattice imaging technique as a tool for structural investigation has been critically evaluated.
\end{abstract}

Keywords. High resolution electron microscopy; silicon carbide; lattice imaging; structural investigation; C-periodicity; stacking sequence; polytype in $\mathrm{SiC}$.

\section{Introduction}

Lattice imaging in the electron microscope has emerged in the recent past as an important high resolution electron microscopy (HREM) technique for the study of ultramicrostructure of crystals. The most valuable capability of HREM concerns the study of local structural variations arising due to non-stoichiometry, stacking faults, etc. Further, it provides an opportunity to study the crystallography of materials directly in the real space compared to available information from diffraction techniques which are in reciprocal space. A number of mixed layer compounds, minerals and complex oxides have been studied by this technique (Van Landuyt et al 1973; Van Landuyt et al 1974; Cowley and Iijima 1972; Allpress and Sanders 1973; Buseek and Iijima 1974). Lattice imaging technique has been extended to two-dimensional crystal structure images by Allpress (1970), Iijima (1971) and lijima et al (1973) and others who showed that image contrast could be directly correlated with structural details. However, the utility of this fascinating technique in structure determination is limited to only those materials which have high thermal stability under the impact of electron beam and have preferably large cell parameters. SiC is one such material which is known to crystallize into several polytype structures of extremely large periodicities.

In $\mathrm{SiC}$, usually large period cells consist of regular stackings of unit cells of more common structure types like $6 \mathrm{H}, 15 \mathrm{R}, 4 \mathrm{H}$ and $21 \mathrm{R}$ which are periodically interrupted by stacking faults (Verma and Krishna 1966). Structures of large c-periodicities are thus generated. Therefore, it has become a common practice to describe any large period structure in terms of stackings of smaller unit cells. The utility of low resolution lattice images has already been established in the structural investigations of SiC structures of large cell parameters (Dubey and Singh 1978; Singh and Singh 1980; Singh et al 1981; Yessik et al 1975). The information available from one-dimensional lattice images is able to reduce the number of probable structures drastically and enables one to determine the correct structure. 
It has been shown recently by Jepps et al (1979) that two-dimensional high resolution pictures are capable of revealing directly the stacking sequence of $\mathrm{Si}-\mathrm{C}$ double layers. $\mathrm{A}$ common feature of most of the lattice imaging work reported in literature has been that they either involve intuitive interpretation or require $n$-beam image contrast calculations which require the knowledge of crystal structure and experimental parameters like foil thickness, defocus etc which are not easily known. During the present investigation a different approach has been adopted in the sense that crystals are first studied by $x$-ray diffraction and then by HREM technique so that the structural inferences drawn from the lattice images may be verified by establishing a match between observed and calculated $x$-ray intensities.

An attempt has been made to determine unknown $\mathrm{SiC}$ polytype structures with the help of lattice imaging which also provides an opportunity for critical evaluation of this technique in structural investigations.

\section{Experimental details}

The structures of $\mathrm{SiC}$ are often found in syntactic coalescence and those of large periodicities are usually in the form of very thin lamellae and it becomes very difficult to get even minute pieces of a crystal which is exclusively a large period structure. Therefore, samples suitable for electron microscope examination are prepared usually by crushing the coalesced crystals into powder form and dispersing the tiny fragments on a holey carbon grid. The iattice images have been taken on JEM $100 \mathrm{CX}$ electron microscope. The most common cleavage plane in $\mathrm{SiC}$ is (0001): However, cleavage parallel to prismatic planes $\{11 \overline{2} 0\}$ is also found though less frequently and thin foils produced due to such cleavages are found suitable for lattice resolution studies.

As all the SiC structures are built up by three-dimensional network of tetrahedrally bonded $\mathrm{Si}$ and $\mathrm{C}$ atoms, they can be described by stacking sequence of either $\mathrm{SiC}$ tetrahedra or $\mathrm{SiC}$ double layers in terms of close-packing of equal spheres. Figure 1 shows six-layered hexagonal structure $(6 \mathrm{H})$ as viewed along $b$-axis. $\mathrm{Si}$ or $\mathrm{C}$ atoms

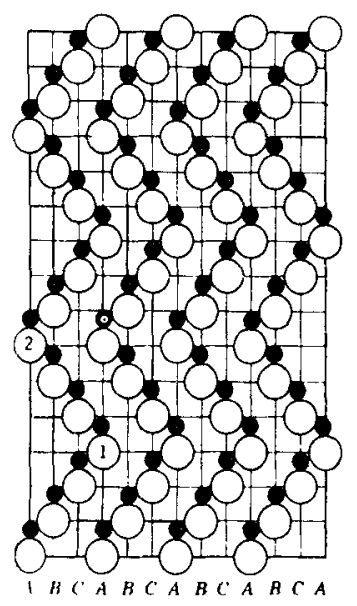

Figure 1. Zigzag sequence of $\mathrm{Si}$ and $\mathrm{C}$ atoms in the $(1120)$ plane of the $6 \mathrm{H}$ polytype of $\mathrm{SiC}$. 
corresponding to a cyclic sequence of layers $A \rightarrow B \rightarrow C$ and those in anticyclic sequence $A \rightarrow C \rightarrow B$ lie in the tetrahedral face planes $(\{10 \overline{1} 2\}$ and $\{10 \overline{1} \overline{2}\}$ in $6 \mathrm{H}$ structure) which are mutually rotated by $\pi$. Since all the polytype structures consist of some combination of cyclic and anticyclic sequence of layers, the $\mathrm{Si}$ or $\mathrm{C}$ atoms always lie on either of the tetrahedral face planes which are inclined equally at $109^{\circ} 28^{\prime}$ with $c$-axis. That means $\{10 \overline{1} l\}$ and $\{10 \overline{1} \bar{l}\}$ planes inclined at this angle with $c$-axis or very near to this in any polytype structure have high reticular density in different parts of the unit cell depending on the layer sequence. Of course the basal planes $(000 l= \pm n)$ for any $n H$ or $n R$ polytype also have high reticular density making these reflections invariably strong.

Now in a zone axis orientation if the objective aperture allows reflections of a twodimensional reciprocal lattice net, the resulting image is a crude projection of the structure along the zone axis. When the incident beam is tilted to place the optic axis midway between $000 \mathrm{l}$ and $10 \bar{l} \mathrm{l}$ rows so as to allow equivalent reciprocal lattice vectors through the aperture, crossed lattice image may be formed from reflections of high reticular density planes viz basal stacking planes and tetrahedral face planes. Due to thickness variation of the sample the distribution of diffracted intensity of the allowed beam varies from one place to another giving rise to images of varying sharpness in terms of relative dominance of basal and inclined fringes. Optimum conditions are searched when chevron-shaped contrast presumably representing reticular density of tetrahedral face pair in projection is achieved. These chevrons along with basal fringes corresponding to individual stacking planes may enable one to uniquely read the stacking sequence of the entire unit cell.

\section{Two-dimensional lattice images}

\section{$3.16 \mathrm{H} \mathrm{SiC}$}

Let us consider the structure image correlation in a simple case of $6 \mathrm{H}$ whose structure is fully known. Figures $2 \mathrm{a}, \mathrm{b}$ show a two-dimensional lattice image taken under tilted beam illumination along with the diffraction pattern. The main features of the lattice image are: (i) the $c$-period blocks $(1.5 \mathrm{~nm})$ of $6 \mathrm{H}$ structure consist of two parts appearing in distinct dark and light contrast. The two parts are of equal width in comparatively thinner regions (top portion of the image) while at other places respective widths vary becoming $1: 2$ at the bottom portion, (ii) the chevron-shaped fringes in the two parts of

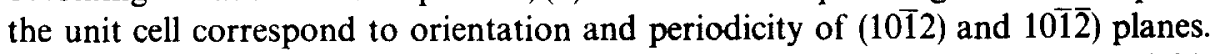

These features may be understood in terms of contributions of the individual diffracted beams giving rise to a sinusoidal distribution of the charge density in the image plane. The wave fronts of this sinusoidal distribution are parallel to those lattice planes that may be supposed to produce the diffracted beam by reflection and its wavelength is equal to the $d$-spacing of these planes. Figure 3 shows the traces of contributing (10ill) planes which also represent the wavefronts of the sinusoidal charge distribution in the image. It may be noted that all the wavefronts coincide along equallyspaced lines parallel to $b$-axis which intersect the $a$-axis at spacings of $3.08 \AA$ (a-parameter). Depending on the phase of the component sinusoidal distributions superposed on these points, pronounced dotted contrast should occur along lines perpendicular to $c$-axis at intervals of $c$-repeat period $(1.5 \mathrm{~nm})$ as is seen in figure 4 . Taking into account the wavefronts of the relevant $(000 l)$ planes also, it can be realized 

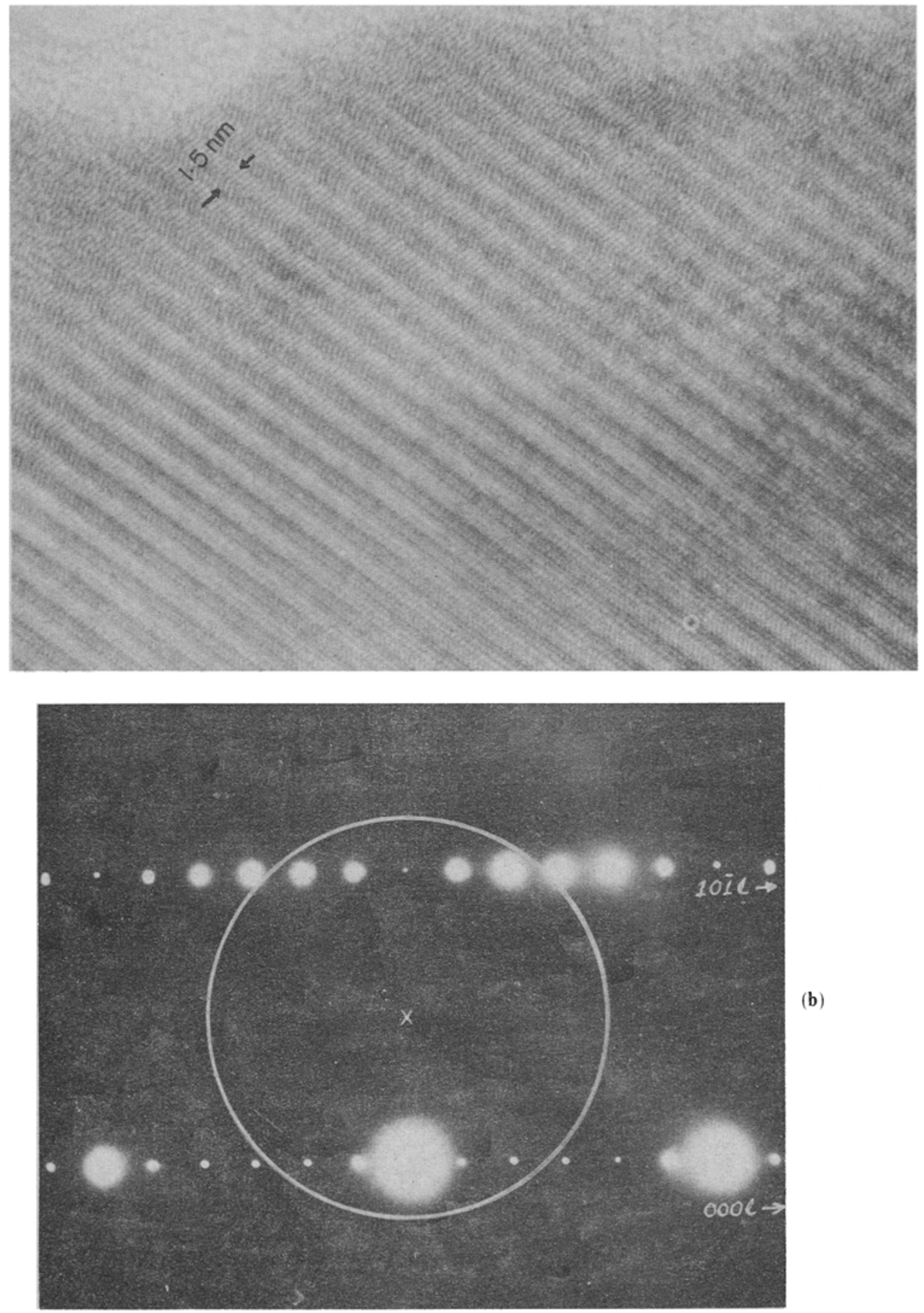

(b)

Figure 2. a. Tilted illumination lattice image of a $6 \mathrm{H} \mathrm{SiC}$. The beam is tilted midway between $10 \overline{10}$ and $000 \mathrm{l}$ so that $10 \overline{1} 2$ and $501 \overline{2}$ reflections are included in the aperture. The inclined fringes forming chevrons are parallel to $(10 \overline{1} 2)$ and $(10 \overline{1} \overline{2})$ planes $(d$ spacing $\approx$ $0.25 \mathrm{~nm}$ ). b. The corresponding diffraction pattern showing the position of optic axis by a cross and objective aperture by circle. 


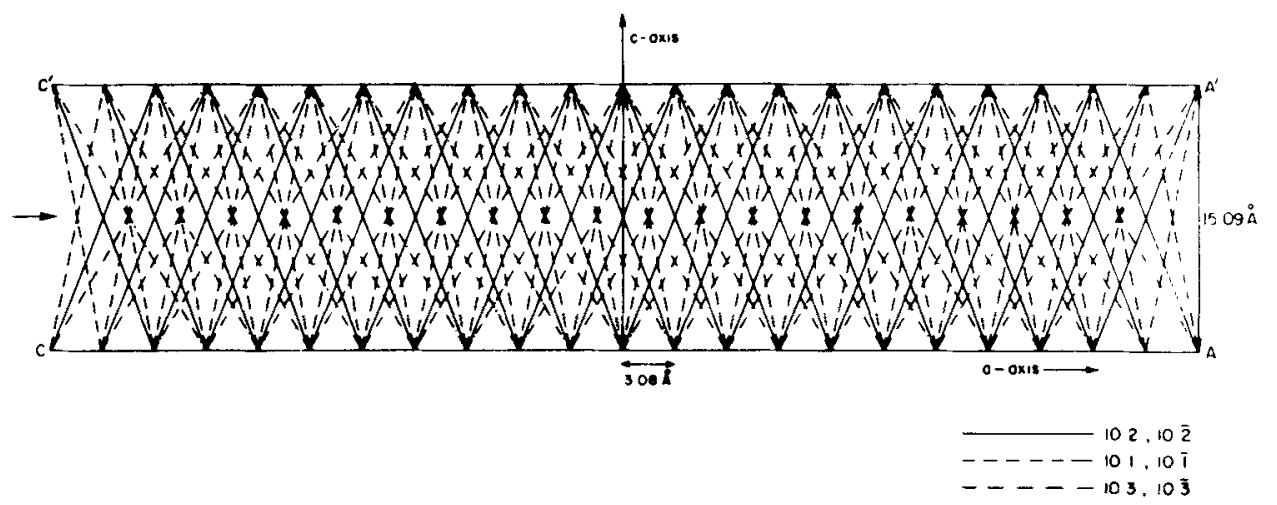

Figure 3. Traces of some 10̄ $l$ lattice planes of the $6 \mathrm{H}$ polytype whose Bragg reflected beams pass through objective aperture. Traces of $(000 l)$ planes have not been drawn to avoid overcrowding.

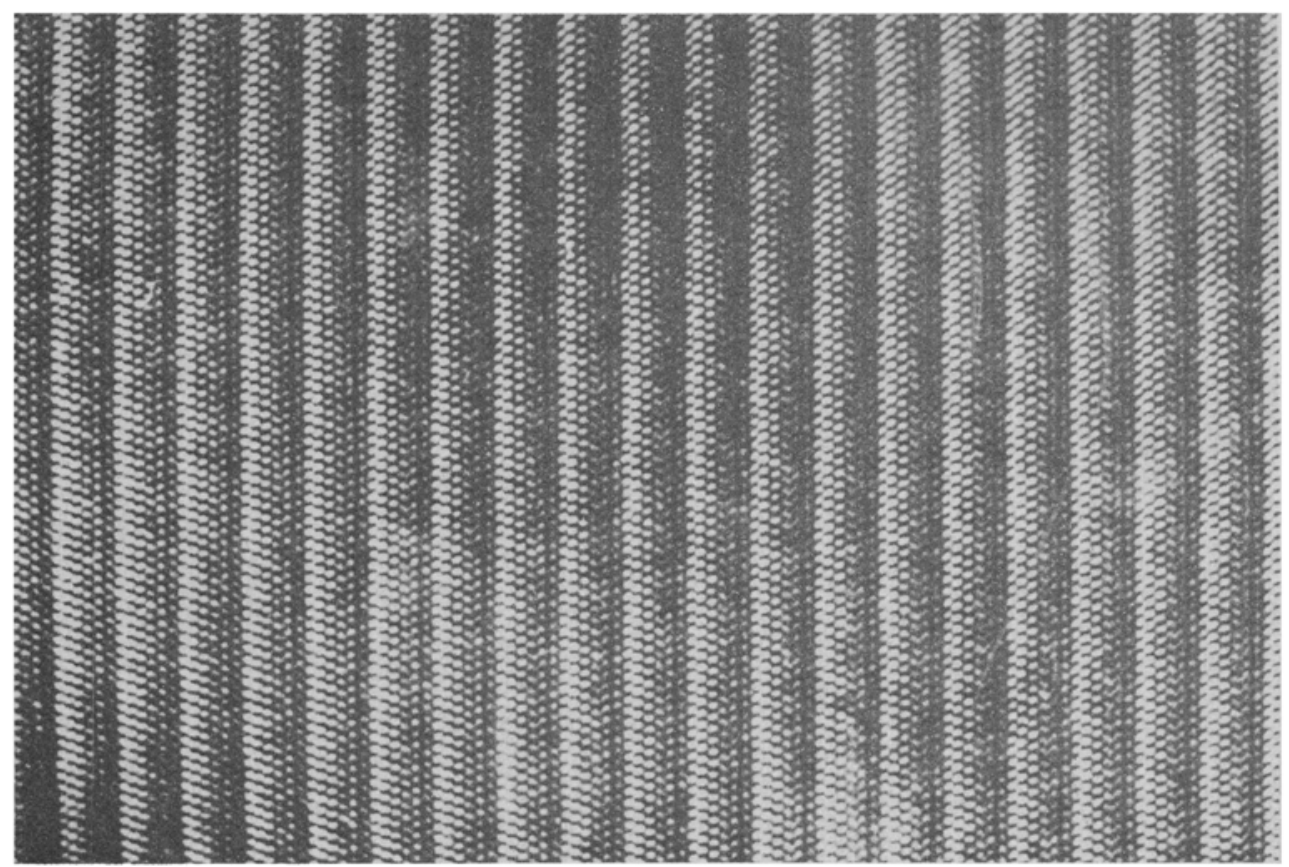

$1.5 \mathrm{~nm}$

Figure 4. Tilted beam lattice image of $6 \mathrm{H} \mathrm{SiC} \mathrm{taken} \mathrm{under} \mathrm{optimum} \mathrm{condition} \mathrm{showing}$ pronounced dotted contrast of both the sets of fringes (basal as well as inclined).

that superposition of wavefronts to a lesser degree also takes place along lines normal to $c$-axis at intervals of interlayer separation $(0.25 \mathrm{~nm})$ throughout the unit cell resulting in subsidiary fringes. Therefore fringes representing fundamental interlayer separation along $c$-axis may be obtained under appropriate conditions provided the instrumental resolution of the microscope allows it. Figure 5 shows these fringes in a bright field 


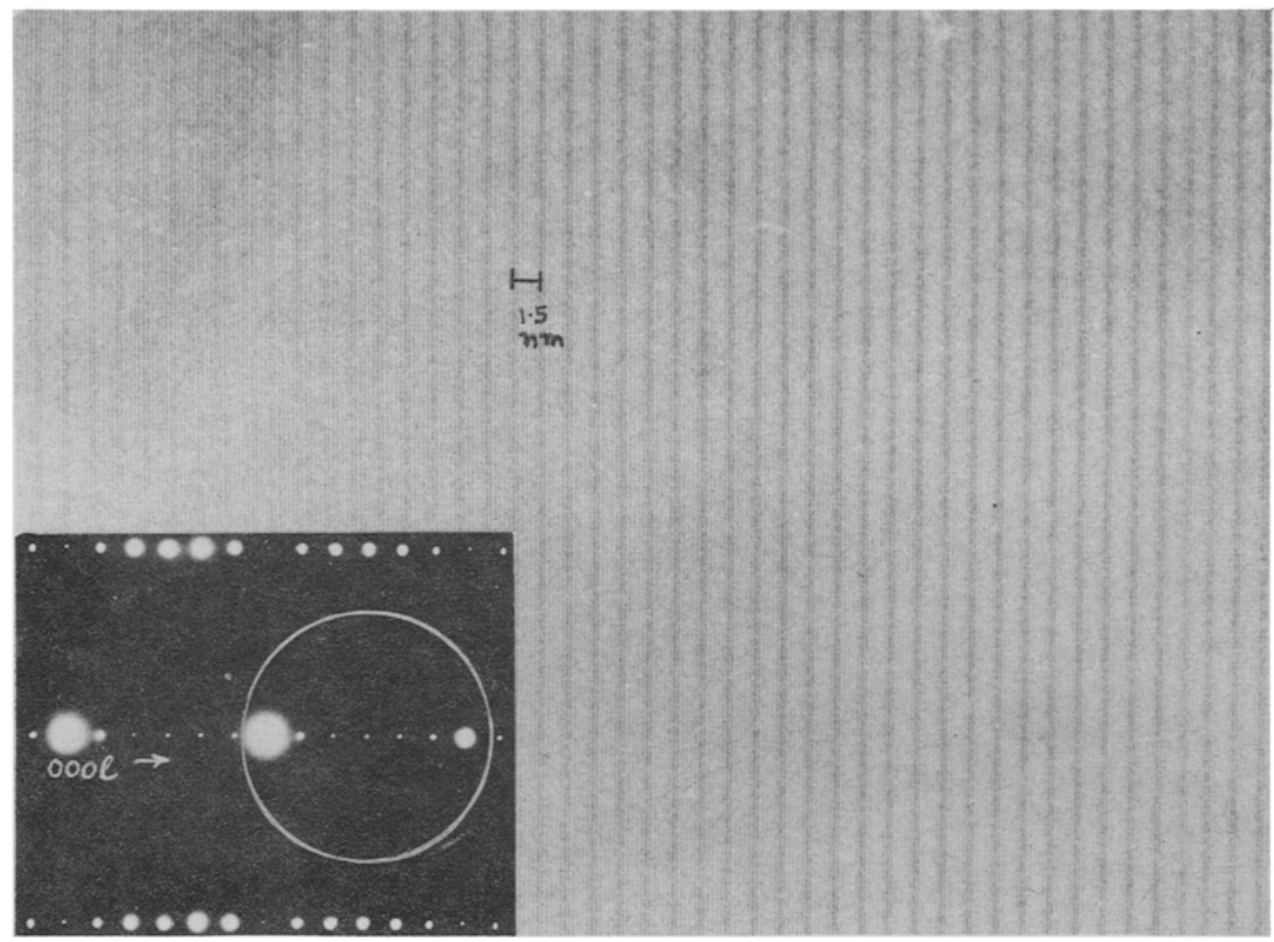

Figure 5. Bright field lattice image showing resolution of $(0006)$ planes (representing interlayer spacing $\sim 0.25 \mathrm{~nm}$ ) in $6 \mathrm{H} \mathrm{SiC}$. Fringe modulations at $1.5 \mathrm{~nm}$ periodicity characterize the $c$-repeat period. The corresponding diffraction pattern is shown as inset.

image formed by symmetrical $000 \mathrm{l}$ reflections and they can as well be obtained from a dark field image formed by $10 \overline{1} l$ reflections. In tilted beam illumination pictures, these fringes can appear only under special experimental conditions. Out of these subsidiary fringes in tilted beam pictures which divide the unit cell into six parts, one at the middle is expected to be comparatively strong as can be seen from the traces of $(10 \overline{1} l)$ planes in figure 3. This is because the $10 \overline{1} l$ reflections are stronger than $000 l$ reflections which appear only due to double diffraction.

Figure $2 \mathrm{a}$ shows a tilted beam image of a $6 \mathrm{H}$ foil where all the linear fringes of $0.25 \mathrm{~nm}$ periodicity do not appear while chevron-shaped fringes of almost the same periodicity are in good contrast and well resolved. If chevron-shaped fringes arise due to dominance of $10 \overline{1} 2$ and $10 \overline{1} \overline{2}$ reflections, one would expect a cross grating pattern of the two sets of fringes. The chevron-shaped contrast can be understood only in terms of crude Fourier projection of the structure along $b$-axis because the image is formed by a limited of reflections of this zone. As can be seen from structure model (figure 1) each plane of the family $(10 \overline{1} 2)$ in one half of the unit cell and that of $(10 \overline{1} \overline{2})$ in the other half consist of a closely-spaced pair of silicon and carbon planes. In a crude projection along $b$-axis these pairs of Si-C atomic planes project forming chevrons of certain width. Since atomic resolution in the used experimental set-up was out of question, the chevron-shaped contrast bears a close correlation with the projected charge density of the closely-spaced $\mathrm{SiC}$ planes. 
The relative dominance of linear and inclined fringes in the image depends on the distribution of diffracted intensity, which may vary from position to position depending on thickness. Optimum conditions may be looked for, when both the sets of fringes are reasonably pronounced. Figure 4 shows an image taken under optimum condition.

Now the layer sequence of the $6 \mathrm{H}$ structure can be read from chevron-shaped fringe of figure 2a. In Zdhanov notation, the layer sequence read from the upper part of the image is 33 while that from the lower part is 24 . This variation arising due to thickness changes introduces uncertainty in the structure which can be resolved only by confirming the structure either by establishing a match between observed and calculated $\mathrm{x}$-ray intensities or between observed and calculated image contrast. In contrast, the linear fringe modulations at spacings of $c$-periodicity $(1.5 \mathrm{~nm})$ are not affected with respect to their periodicities by thickness variations to the same extent as chevron shaped fringes are. As a result, the derived structural information remains ambiguous unless confirmed by other means. Therefore, we have made use of HREM (twodimensional lattice imaging) and $\mathrm{x}$-ray diffraction as a complimentary tool in solving the structural details of a new polytype $411 \mathrm{R}$ of $\mathrm{SiC}$ and its intergrowth structures which is described below.

\section{A new polytype of $\mathrm{SiC} 411 \mathrm{R}$ and its intergrowth structures}

\subsection{X-ray diffraction characteristics}

A bluish black crystal of platelet shape $(3 \times 2 \times 0.35 \mathrm{~mm})$ having well-developed basal faces $\{0001\}$ was chosen from a batch of $\mathrm{SiC}$ crystals grown from vapour phase (Lely's method). The $c$-axis oscillation photographs in two ranges of oscillation of this crystal (figures 6a, b) show spots of a 137-layered polytype superimposed over faint streak along $10 \overline{1} l$ reciprocal lattice row. Unique identification of the polytype was not possible at this stage because spots on one side of the zero-layer line are absent due to absorption. This indicates that high period polytype exists in a thin lamellar form on the platelet of $6 \mathrm{H}$ structure and it was not possible to isolate its smaller piece for detailed $\mathrm{x}$-ray examination. A detailed investigation of this crystal was subsequently done by lattice imaging in symmetric bright field and tilted illumination modes with twin purpose of deriving the structure as well as assessing the capability of chevron-shaped fringes for structural investigations.

\subsection{Lattice imaging studies}

After examining the crystal by $x$-ray diffraction, suitable samples for electron microscopic investigation were prepared by crushing the crystal, as discussed earlier. A thin crystal flake was brought in zone axis orientation giving hoil reflections as shown in figure $7 \mathrm{a}$. Figure $7 \mathrm{~b}$ is a bright field image obtained from symmetrical $000 \mathrm{l}$ reflections. The lattice image reveals a superperiodicity of $145.5 \mathrm{~nm}$ (figure $7 \mathrm{~b}$ ). Each superperiod consists of a sequence of four blocks of smaller periods of periodicities $34.5,33,34.5$ and $43.5 \mathrm{~nm}$ respectively. A dark field picture taken with only $101 \mathrm{l}$ reflections also reveals similar pattern of periodicities. The same pattern of fringes occur over an extended area in the sample and figure $7 \mathrm{~b}$ shows only a part of it. It may be seen that most of the fringe spacing correspond to $6 \mathrm{H}$ periodicity of $1.5 \mathrm{~nm}$. The formation of four sub-periods and the superperiod essentially takes place due to insertion of faults in 


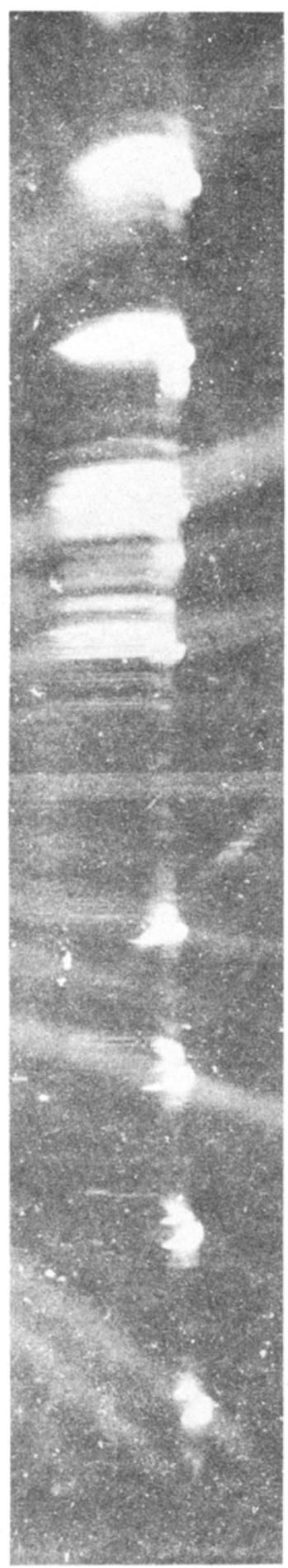

(a)

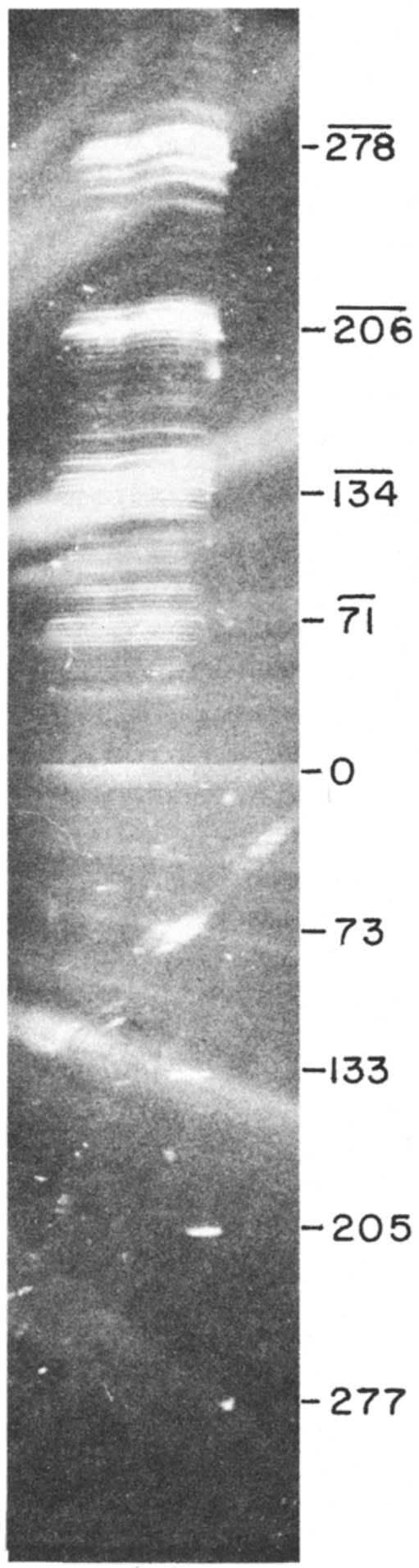

(b) 
a regular sequence of the $6 \mathrm{H}$ matrix. A periodicity as large as $145.5 \mathrm{~nm}$ cannot be normally detected by $\mathrm{X}$-ray diffraction and therefore the faint streak in the $\mathrm{x}$-ray pattern can be easily assigned due to this super-periodicity. The periodicity revealed by $x$-ray diffraction (figure $6 \mathrm{~b}$ ) corresponds to the periodicity of $34.5 \mathrm{~nm}$ which occurs twice in the superperiod block.

A two-dimensional lattice image (figure $8 \mathrm{a}$ ) was taken under tilted beam illumination by including encircled reflections (figure 8 b). To show at least one complete superperiod blook this image has not been sufficiently magnified to clearly show twodimensional features. Pictures were taken at different defocus values and one of them showing chevron-shaped fringes in good contrast is shown in figure 8a. As discussed earlier, the chevron-shaped fringes may be assumed to represent the layer stacking sequence of the structure. The magnified pictures of the three sub-units of the superperiod from figure $8 \mathrm{a}$ are shown in figures $9 \mathrm{a}, \mathrm{b}, \mathrm{c}$. The stacking sequence read from this image for $34.5 \mathrm{~nm}$ block (figure 9a) in Zhdanov notation is $3223332223(33)_{2}$ $433332(33)_{9} 22(33)_{4}$. However, the stacking sequence read in this fashion is not unambiguous in the sense that some of the asymmetric units like 43 (marked in figure 9 a) are not manifested very clearly. The stacking sequence read from pictures taken at a slightly different defocus value from figure 8a reveals a different stacking sequence. It is this extreme sensitiveness of these fringes which introduce uncertainty in the structural information derived from them. Therefore, lattice image information must be confirmed by other means.

The above layer sequence read from the lattice image has $n_{+}-n_{-} \neq 3 r$ (where $n_{+}$ represents the total number of layers in the cyclic sequence, $n_{-}$that of anticyclic sequence in the structure and $r$ is any positive or negative integer including zero) and thus it corresponds to rhombohedral lattice and has to be repeated thrice to complete the hexagonal unit cell. The complete structure is given as:

$$
\left[\begin{array}{lll}
3223332223(33)_{2} & 433332(33)_{9} & 22(33)_{4}
\end{array}\right]_{3} \text {. }
$$

Thus the polytype will be identified as $411 \mathrm{R}$ if this structure is correct. It is sufficient to compare the calculated and observed intensities of $10 \overline{1} /$ reflections alone to determine the correctness of a proposed structure of any SiC polytype (Verma and Krishna 1966; Dubey and Singh 1978; Singh and Singh 1980; Singh et al 1981; Yessik et al 1975; Jepps et al 1979; Ramsdell 1944). Accordingly, the intensities of $10 \overline{1} l$ reflections were calculated for the above structure and corrected for Lorentz and polarization factors. Though the full range of $10 \overline{1} l$ reflections is not experimentally available due to reasons discussed earlier, the match between the calculated and observed $x$-ray intensities for the available reflections is quite good. This amply confirms the structure which was already read from the two-dimensional image with some reliability. The atomic positions of $\mathrm{Si}$ and $\mathrm{C}$ atoms can be derived from the Zhdanov symbol of the 411R structure.

Figure 6. a. The $10 \tilde{I} !$ reflections as recorded on a $15^{\circ}, c$-axis oscillation photograph of the parent $\mathrm{SiC}$ crystal. Polytype reflections along $c^{*}$ are superimposed over faint streak $(\times 7)$. b. The $10 \overline{1} l$ reflections as recorded on a $15^{\circ}, c$-axis oscillation photograph of the same crystal in a different range of oscillation. Spots are better resolved showing a periodicity of $137 \mathrm{H}$ or $411 \mathrm{R}$ polytype. Spots on one side of the zero-layer line are absent due to adsorption $(\times 7), \mathrm{CuK} \alpha$ radiation. 


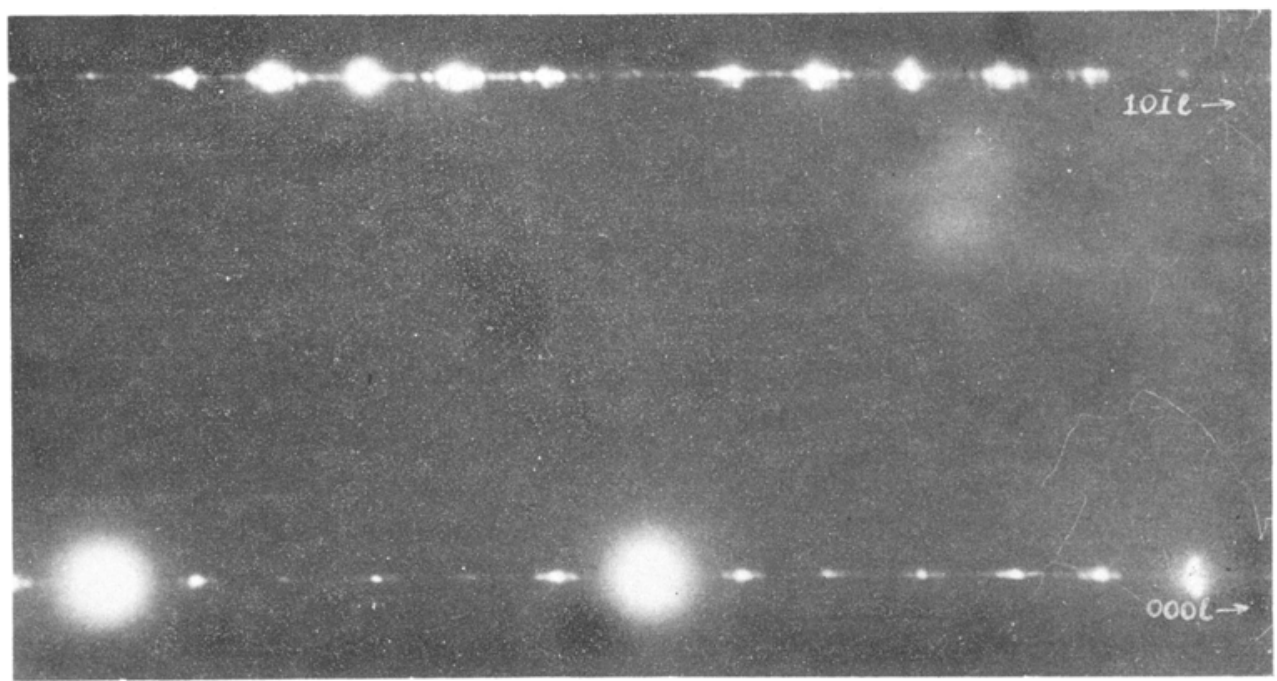

(a)

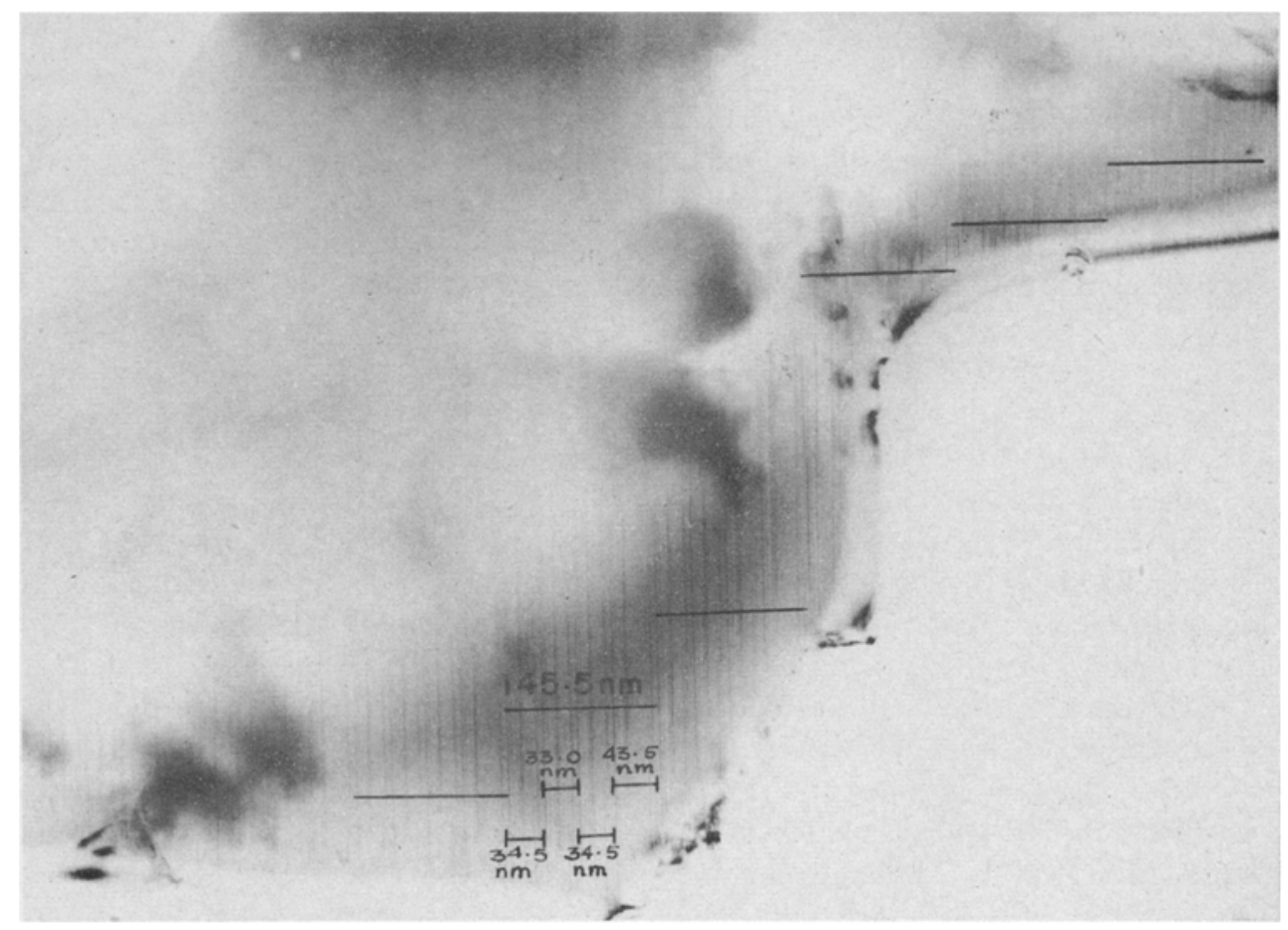

(b)

Figure 7. a. Electron diffraction pattern showing the hoil reciprocal lattice net of the same crystal as in figure 6 b. b. A bright field lattice image, taken with symmetrical $000 /$ reflections. Super-period blocks of $145.5 \mathrm{~nm}$ periodicity are marked. Each block consists of intergrowth of $34.5,33,34.5$ and $43.5 \mathrm{~nm}$ blocks. The $34.5 \mathrm{~nm}$ blocks correspond to periodicity inferred from $x$-ray diffraction. 


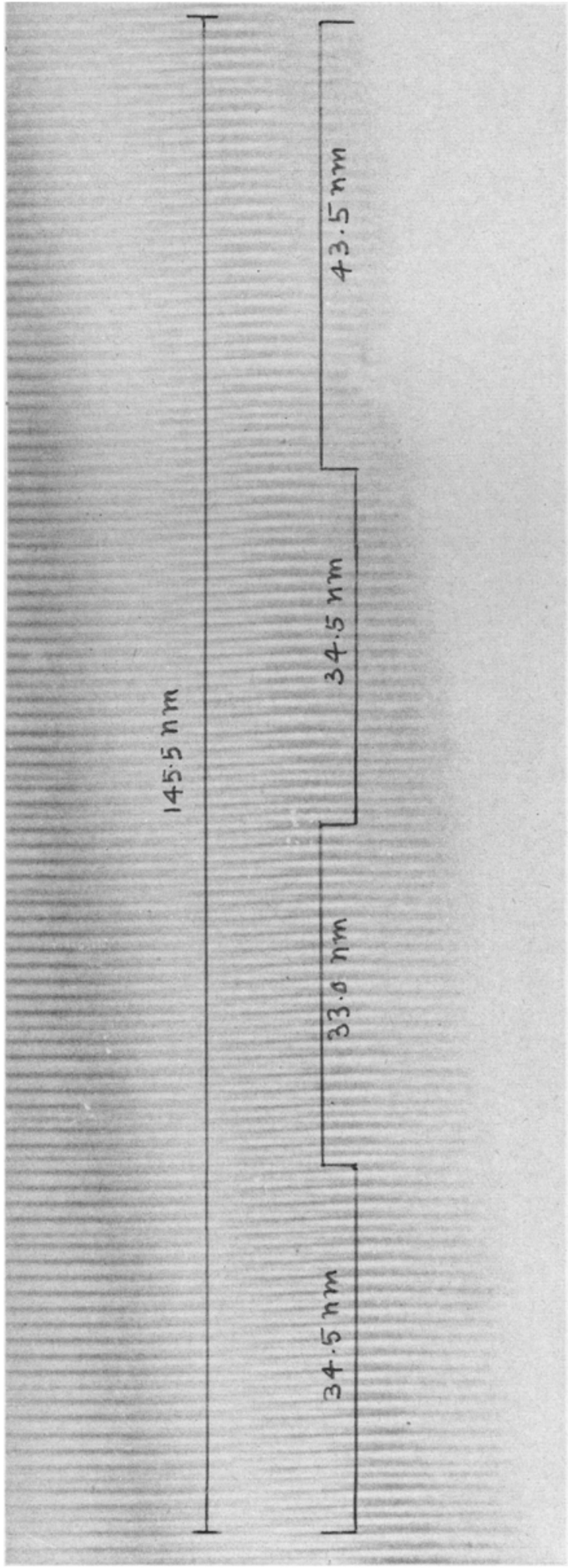




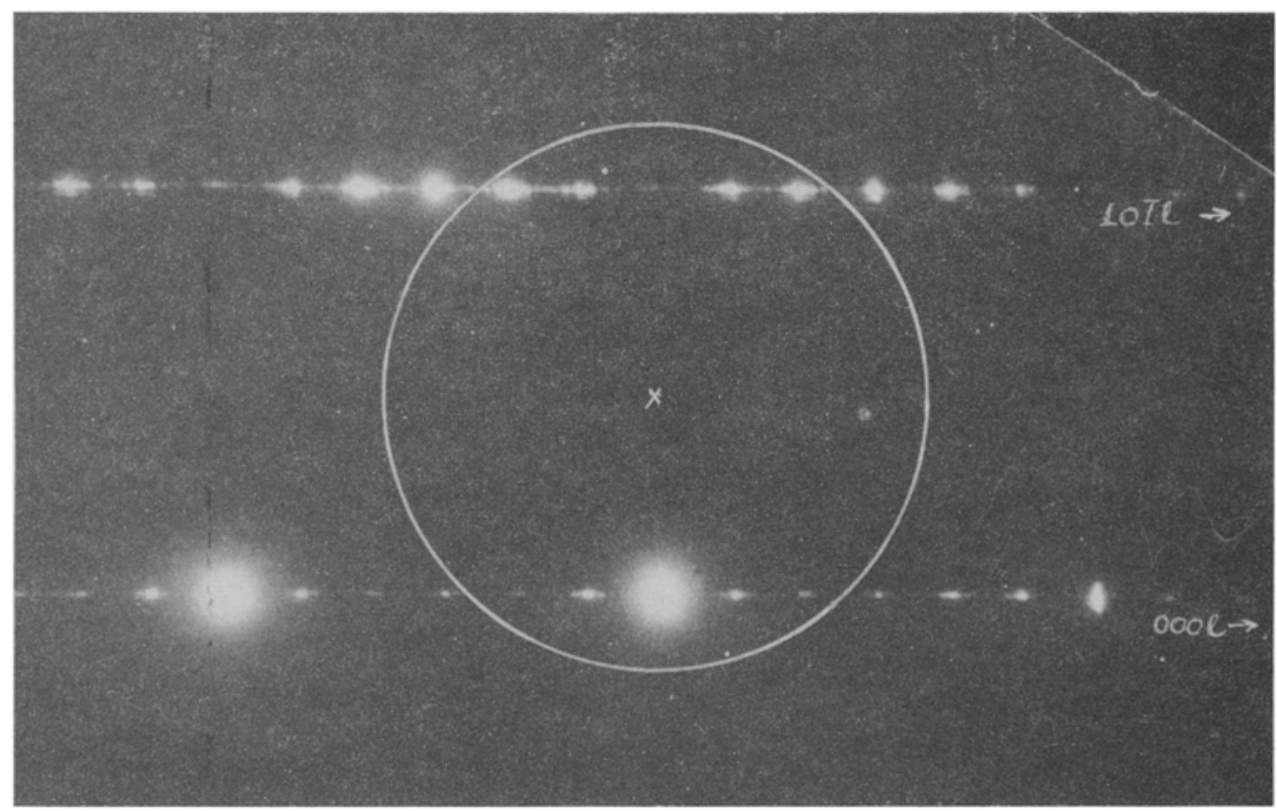

(b)

Figure 8. a. Two-dimensional lattice image taken under tilted beam illumination of the same crystal. Objective aperture symmetrically includes $000 l$ and $10 \bar{i} \hat{l}$ reflections. b. Corresponding diffraction pattern, position of aperture and optic axis are marked.

\subsection{Intergrowth structures of $411 R$}

It has been seen from figures $7 \mathrm{~b}$ and $8 \mathrm{a}$ that the super-period structure is built up of subunits of periodicity 43.5 and $33 \mathrm{~nm}$ in addition to those of $411 \mathrm{R}$. Since it has been possible to derive the layer sequence of $411 \mathrm{R}$ units from the chevron-shaped fringes, the structures of other intergrowth units can also be derived with reliability.

It can be seen from figure $9 \mathrm{c}$ that the layer sequence in 43.5 block is similar to that of $411 \mathrm{R}$ except that it contains a block of successive 15 fringes of $6 \mathrm{H}$ periodicity in place of nine such fringes in $411 \mathrm{R}$ unit cell. On the other hand, the $33 \mathrm{~nm}$ wide block has again similar layer sequence as in $411 \mathrm{R}$ except that it has eight successive fringes of $6 \mathrm{H}$ periodicity in place of nine in $411 \mathrm{R}$ unit (figure $9 \mathrm{~b}$ ).

\subsection{Super-period structure}

After decoding the layer sequence of constituent units, which intergrow coherently to form a super-period structure, one can write the full layer sequence of this structure in Zhdanov notation as

$$
\begin{aligned}
& {\left[\{ 3 2 2 3 3 3 2 2 2 3 ( 3 3 ) _ { 2 } 4 3 3 3 3 2 ( 3 3 ) _ { 9 } 2 2 ( 3 3 ) _ { 4 } \} \left\{3223332223(33)_{2}\right.\right.} \\
& \left.\begin{array}{lllll}
433332(33)_{8} & \left.22(33)_{4}\right\} & \left\{3223332223(33)_{2}\right. & 433332(33)_{9} & 22(33)_{4}
\end{array}\right\} \\
& \left.\left\{3223332223(33)_{2} 433332(33)_{15} 22(33)_{4}\right\}\right] \text {. }
\end{aligned}
$$

This represents a rhombohedral structure having $1734 \mathrm{SiC}$ double layers in the 


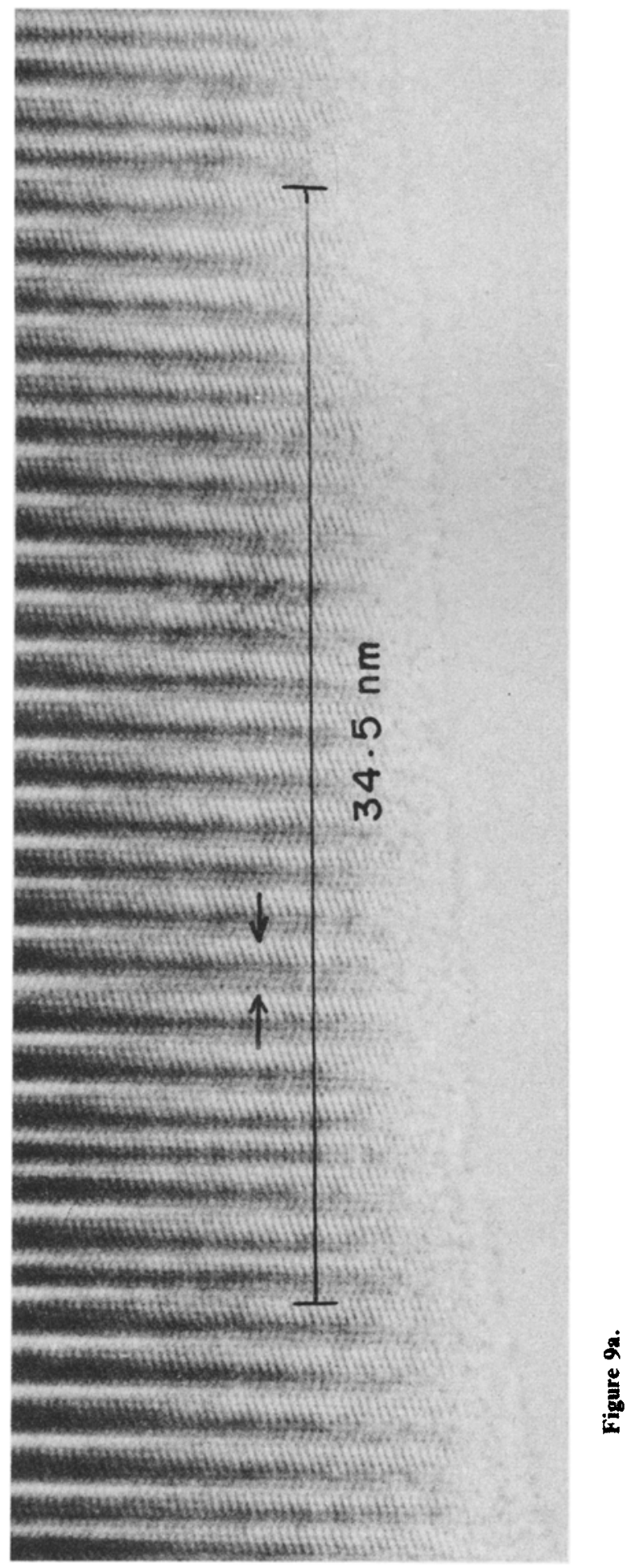




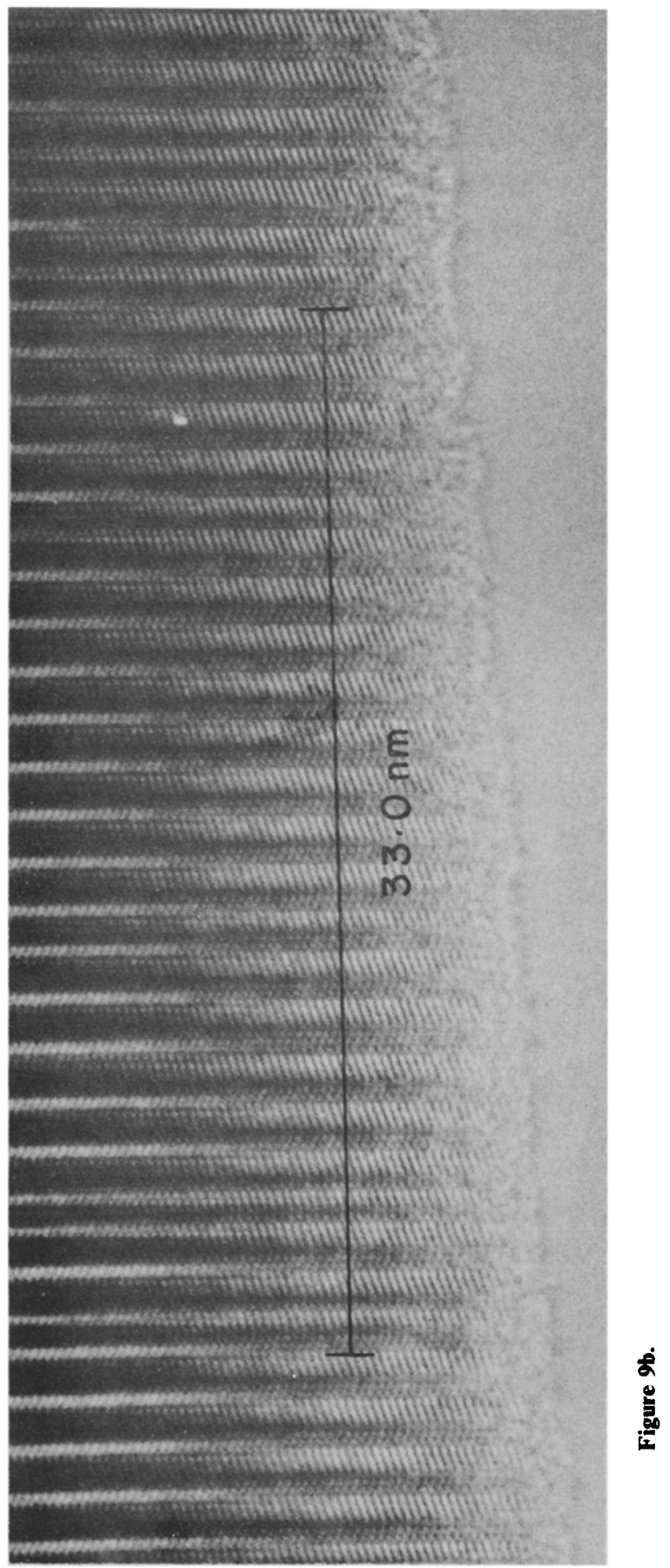




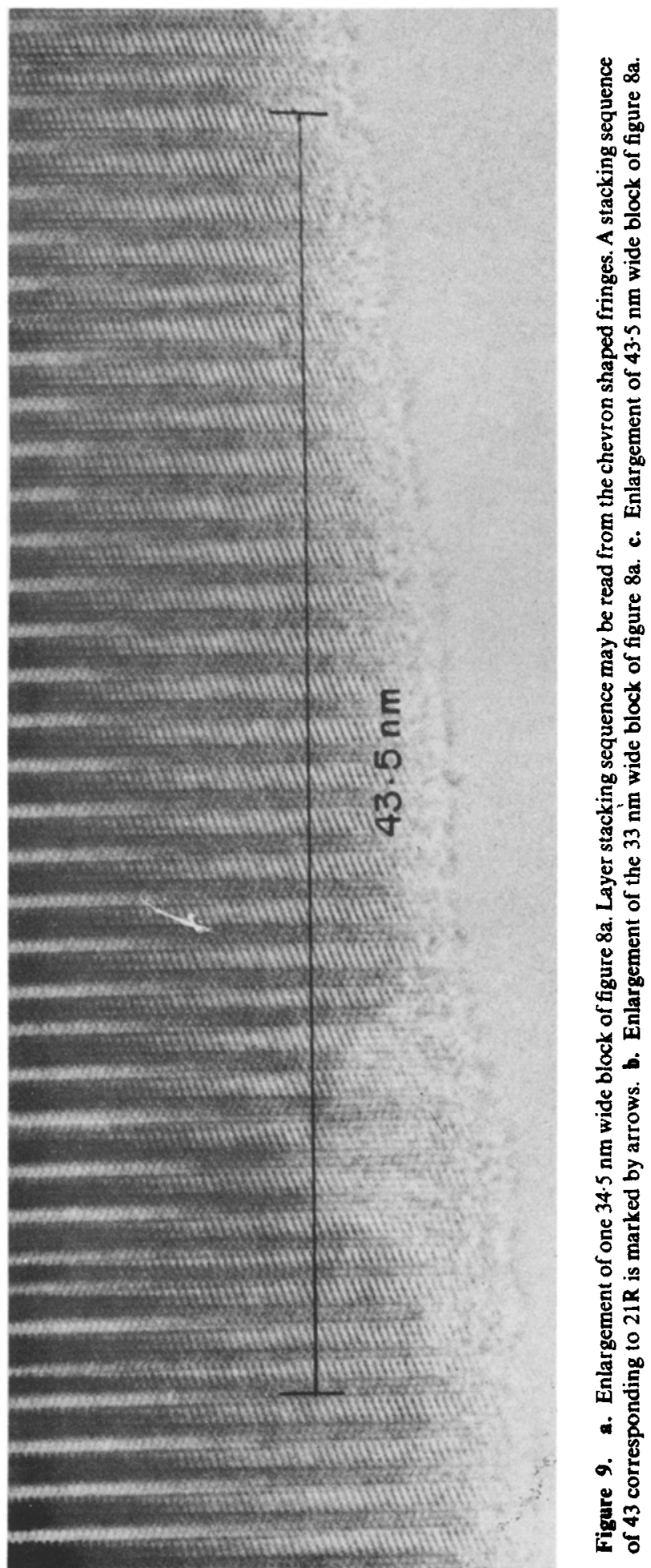


hexagonal unit cell. Therefore, it is identified as $1734 \mathrm{R}$ and the complete structure is derived by repeating the above Zhdanov sequence three times.

\section{Discussion}

It has been seen from the above examples that tilted beam two-dimensional lattice images taken under suitable conditions contain direct information about the stacking sequence of layers in $\mathrm{SiC}$ structures. In $6 \mathrm{H} \mathrm{SiC}$ by including highly excited $10 \overline{1} 2$ and $10 \overline{1} \overline{2}$ reflections in the image formation, which are Bragg reflected from high reticular density planes forming chevron shaped surfaces in the structure, it is possible in principle to read the layer stacking sequence of the structure from chevron shaped fringes. However, chevron-shaped fringes simulating the layer stacking sequence are extremely sensitive to experimental parameters. As a result of this, the derived structural information remains ambiguous unless confirmed by other means. The reason for this state of affairs lies in the limited number of reflections used for obtaining these pictures.

For a real simulation of stacking reversal of layers we need details at the level of $0.25 \mathrm{~nm}$ in the image. This in turn requires inclusion of additional higher orders of diffraction spectra in the image formation, which are excluded, to avoid image deterioration due to spherical aberration. Therefore, a two-dimensional image will resemble a crude projection of the structure only when the foil is thin enough to behave as a phase grating and the image is taken under Scherrer defocus condition.

In contrast to chevron-shaped fringes, the linear fringes normal to $c$-axis arise essentially due to interference of different reflections of individual reciprocal lattice rows along $c^{*}$, like 0001 or $10 \overline{1} l$. An image formed by reflections of only one reciprocal lattice row can give the structure projected along a line. This gives rise to fringe modulations at spacings of $c$-periodicity of common polytypes which is of the order of $1 \mathrm{~nm}$ or more. Since the order of diffraction maxima along $c^{*}$ allowed through the objective aperture is sufficient to give spatial frequencies in the image up to $0.25 \mathrm{~nm}$, the series truncation errors are comparatively less and fringe modulations at the level of $1 \mathrm{~nm}$ or more become comparatively less sensitive to experimental parameters. Thus though the structural information available from one-dimensional fringes contains less details it is reliable. Therefore, wherever possible a combination of linear fringe spacings and chevron shaped fringes should be utilized to derive the structural information.

In $411 \mathrm{R}$ polytype let us consider only linear fringe spacings along $c$-axis. The unit cell contains fringe spacings expressed in terms of number of interlayer separation in the following sequence:

$$
5,5,6,4,5,(6)_{2}, 7,6,5,(6)_{9} 4(6)_{4} \text {. }
$$

This sequence may be converted into Zhdanov symbol by converting a 5 layer spacing into 32 or 23, a seven layer spacing into 43 and 34 and those of six and four layer spacing by 33 and 22 respectively. If the number of such spacings in the unit cell are large, the number of most probable structures even after taking a lattice image remains large. A good two-dimensional image in such complicated cases can lead straightaway to the correct structure, as shown by the present example. The structure determination of this polytype highlights the ambiguity in deriving structural information from lattice images unless properly confirmed by other means.

The details of the nature of intergrowth revealed by lattice imaging are characteristic 
of the power of this technique. In X-ray diffraction, characteristic spots of only $34.5 \mathrm{~nm}$ periodicity were observed whereas the real structure was built by regular intergrowth of three sub-units slightly different from each other. Therefore, the average structure observed by $x$-rays is only that of $411 R$. The super-periodicity of $145.5 \mathrm{~nm}$ simply gives rise to faint streaks due to lack of resolution. The nature of intergrowth observed in this example is not an isolated case. Similar intergrowths have also been observed in $150 \mathrm{R}$ and $40 \mathrm{H}$ (Singh and Singh 1980; Singh et al 1981) and this seems to be a general trend connected with the formation of large period polytypes in $\mathrm{SiC}$. This aspect has come to light in greater detail only as a result of lattice imaging studies.

\section{Acknowledgement}

The authors are grateful to Prof. K H Jack of the Crystallography Laboratory, University of Newcastle upon Tyne, UK for providing experimental facility of JEM $100 \mathrm{CX}$.

\section{References}

Allpress J G 1970 J. Solid State Chem. 278

Allpress J G and Sanders J V 1973 J. Appl. Cryst. 6165

Buseck P R and lijima S 1974 Am. Miner. 591

Cowley J M and lijima S 1972 Z. Naturforsch. Tiel A27 445

Dubey M and Singh G 1978 Acta Crystallogr. A34 116

lijima S 1971 J. Appl. Phys. 425891

Iijima S, Cowley J M and Donnay G 1973 Tschermaks. Miner. Petogr. Mitt 20216

Jepps N W, Smith D J and Page T F 1979 Acta Crystallogr. A35 916

Ramsdell L S 1944 Am. Miner. 29431

Singh S R and Singh G 1980 Acta Crystallogr. A36 779

Singh S R, Singh G and Van Tendeloo G 1981 Acta Crystallogr. A37 625

Van Landuyt J, Amelinckx S, Kohn J A and Eckart D W 1973 Mater. Res. Bull. 81173

Van Landuyt J, Amelinckx S, Kohn J A and Eckart D W 1974 J. Solid State Chem. 9103

Verma A R and Krishna P 1966 Polymorphism and Polytypism in Crysials, (New York)

Yessik M, Shinozaki S and Sato H 1975 Acta Crystallogr. A31 764 